\title{
CRENÇAS DOS PORTADORES DE DOENÇA CORONARIANA, SEGUNDO O REFERENCIAL DE ROKEACH, SOBRE O COMPORTAMENTO DE FUMAR ${ }^{1}$
}

\author{
Rosamary Aparecida Garcia Stuchi \\ Emilia Campos de Carvalho ${ }^{3}$
}

Stuchi RAG, Carvalho EC. Crenças dos portadores de doença coronariana, segundo o referencial de Rokeach sobre 0 comportamento de fumar. Rev Latino-am Enfermagem 2003 janeiro-fevereiro; 11(1):74-9.

Estudo observacional, descritivo, que objetivou identificar as crenças segundo a centralidade/perifericidade, e verificou como elas interferem no comportamento de pacientes portadores de coronariopatias, para aderirem ou não ao tabagismo. Utilizamos um dos referenciais de crenças mencionados na literatura, que evidenciou, na análise das entrevistas realizadas com 56 pacientes portadores de doença coronariana, internados em uma instituição hospitalar do Estado de São Paulo, um predomínio de crenças primitivas de consenso zero e uma tendência à centralidade, o que permitiu identificar fatores que dificultam a adesão às recomendações terapêuticas feitas pelos profissionais da saúde e inferir sobre a resistência para mudança de comportamento que tal população possui.

DESCRITORES: coronariopatias, tabagismo

\section{BELIEFS OF PATIENTS WITH CORONARY DISEASE ACCORDING TO ROKEACH'S THEORETICAL FRAMEWORK CONCERNING THE HABIT OF SMOKING}

This is a descriptive observational study that aimed at identifying beliefs according to centrality/periphery by verifying how they interfere in the behavior of patients with coronary diseases regarding their adherence or non-adherence to smoking. The belief-related frameworks mentioned in the literature were used and findings, pointed out from the analysis of the interviews with 56 people with coronary diseases who were inpatients in a hospital in the interior of São Paulo State, showed a prevalence of primitive beliefs with zero consensus and a tendency to centrality that enabled the identification of hiding factors to the adherence to therapeutic recommendations by health professionals as well as inferences concerning the reluctance to behavioral changes presented by the population.

DESCRIPTORS: coronary diseases, smoking

\section{CREENCIAS DE LOS PORTADORES DE ENFERMEDAD CORONARIA, SEGÚN EL REFERENCIAL DE ROKEACH SOBRE EL COMPORTAMIENTO DE FUMAR}

Estudio observacional, descriptivo que buscó identificar las creencias según la centralidad/perifericidad y verificó como ellas interfieren en el comportamiento de pacientes portadores de coronariopatías para convertirse o no en fumadores. Utilizamos unos de los referenciales de creencias, que evidenció, en el análisis de las entrevistas realizadas con 56 pacientes portadores de la enfermedad coronariana internados en una institución hospitalaria del Estado de São Paulo, un predominio de creencias primitivas de consenso cero y una tendencia a la centralidad que permitió identificar factores obstaculizadores para la aceptación de las recomendaciones terapéuticas realizadas por los profesionales de salud e inferir sobre la resistencia para el cambio de comportamiento que dicha población posee.

DESCRIPTORES: coronariopatias, fumadores

\footnotetext{
${ }^{1}$ Este trabalho é parte da dissertação de Mestrado "Crenças dos portadores de doenças coronariana sobre os comportamentos de risco" apresentada à Escola de Enfermagem de Ribeirão Preto da Universidade de São Paulo; ${ }^{2}$ Doutoranda do Programa Enfermagem Fundamental, Professor Assistente da UNIOESTE-PR; ${ }^{3}$ Professor Titular, e-mail: ecdcava@eerp.usp.br. Escola de Enfermagem de Ribeirão Preto da Universidade de São Paulo, Centro Colaborador da OMS para o desenvolvimento da pesquisa em enfermagem
} 
INTRODUÇÃO

A doença cardiovascular (DCV) foi responsável por, aproximadamente, $44 \%$ de todos os óbitos nos Estados Unidos, em 1987, isto é, cerca de duas vezes o número causado por câncer. Mais da metade das mortes por DCV são explicadas pelas cardiopatias coronarianas resultantes da aterosclerose (53\%), principalmente o infarto do miocárdio, angina pectoris e suas seqüelas ${ }^{(1)}$.

No Brasil, as DCV acometem cerca de 300.000 brasileiros ao ano. Sendo uma das principais causas de óbitos (840/dia), ocasionando gastos em assistência médica, implicando maior causa de ocupação de leitos, ou seja, 10,74 dias de internação pelo Sistema Único de Saúde ${ }^{(2)}$.

A doença coronariana ou insuficiência coronária ou coronariopatia, doença isquêmica cardíaca, ocorre quando há um desequilíbrio entre a oferta de oxigênio e as necessidades do coração em relação a esse elemento, para desempenhar suas funções adequadamente. Uma das primeiras manifestações desse desequilíbrio é a dor, em resposta à falta de suprimento/sangue no músculo cardíaco.

Estudos epidemiológicos demonstraram que a doença coronariana e suas complicações estão associadas a uma grande variedade de fatores de risco. $\mathrm{O}$ estudo de Framinghan, nos Estados Unidos, foi o primeiro a descrever os fatores de riscos primários e secundários para coronariopatia ${ }^{(3-5)}$.

Os fatores de risco coronário referem-se às condições capazes de aumentar a suscetibilidade de um indivíduo à morbidade e mortalidade da aterosclerose coronária $^{(3)}$ e podem ser classificados em alteráveis: hipertensão arterial, dislipidemias, tabagismo, diabetes, obesidade, vida sedentária, uso de anticoncepcionais hormonais, estresse emocional, etc. e fatores que não podem ser alterados: idade, sexo, raça, antecedentes familiares da doença coronariana ${ }^{(4)}$.

O controle desses fatores pode ser estendido a todos os grupos que apresentam coronariopatias, seja nas diferentes formas de angina, no infarto ou pós-infarto, antes e após a cirurgia de ponte de safena ${ }^{(6)}$.

O consumo de cigarro tem atingido cifras altíssimas na grande maioria dos países desenvolvidos. Estima-se que existem 1 bilhão de fumantes na população mundial.

No Brasil, os índices demonstram a existência de 3,3 milhões de fumantes. No Estado de São Paulo, $45 \%$ dos homens e $32 \%$ das mulheres são fumantes ${ }^{(7)}$.

Em estudo realizado a respeito do perfil dos pacientes com infarto agudo do miocárdio, envolvendo uma amostra de 45 pacientes, revelou que $80 \%$ dessa amostra constava de pacientes fumantes e ex-fumantes ${ }^{(8)}$.

Das substâncias presentes na fumaça do cigarro, pelo menos quatro foram identificadas e relacionadas como causadoras de danos cardiovasculares: o monóxido de carbono, a nicotina, o benzopireno e os radicais livres ${ }^{(9-}$ 11).

A nicotina e o monóxido de carbono são, dos agentes liberados pelo cigarro, os mais envolvidos na gênese das doenças coronarianas e de suas manifestações ${ }^{(11)}$. Estudos comprovaram que o risco relativo de desenvolver infarto do miocárdio está relacionado ao número de cigarros fumados e varia de 2,1 a 4,0 (média $2,8)$, não sendo reduzido pelo uso de cigarros pobres em nicotina ${ }^{(11-12)}$.

Daí a necessidade de priorizarem-se medidas de prevenção primárias por meio de modificações dos fatores de risco cardiovascular. Freqüentemente, responsabilizamos como determinantes da doença, além dos agentes causais, condições básicas do próprio hospedeiro, o ambiente, e não devemos nos esquecer de que o comportamento de risco está igualmente incluído.

Diversos modelos de pesquisa vêm sendo desenvolvidos para entender o comportamento na área de saúde, entre eles podemos destacar: modelos estruturais, modelos de barreiras situacionais, modelos educacionais e o modelo de crença de saúde.

Apesar dos recentes progressos na ciência e tecnologia, na área da saúde, os indivíduos continuam a adotar comportamentos que, muito freqüentemente, acabam por prejudicar sua saúde a curto, médio e longo prazo $^{(13)}$.

Em nossa prática profissional, atuando junto aos portadores de doença coronariana e com o fator de risco "tabagismo" presente, temos observado que, apesar dos esforços dos profissionais de saúde para minimizar os riscos de um infarto ou reinfarto nessa clientela, muitas vezes esse comportamento preventivo tem sido desconsiderado pela maioria dos indivíduos. Acreditamos que as crenças sejam um dos componentes que possam estar influenciando para a adesão ou não de comportamento que conduzam à promoção da saúde.

Esta pesquisa enfocará apenas o comportamento 
de fumar, tendo, como objetivo, identificar as crenças segundo a centralidade/perifericidade, conforme referencial adotado $^{(14)}$, e verificar como elas interferem no comportamento de pacientes portadores de doença coronariana na adesão ou não às recomendações do profissionais de saúde.

Acreditamos que estudos dessa natureza possam contribuir para a elaboração de estratégias ou programas de educação e prevenção da doença coronariana, levandose em consideração as crenças dos indivíduos com relação ao uso do tabaco.

\section{REFERENCIAL TEÓRICO}

Essa teoria contempla uma explicação do comportamento humano baseado em um sistema de crenças, atitudes e valores. Nesse enfoque, as crenças são as centenas de milhares de declarações (usualmente inferências) que as pessoas fazem a respeito do seu eu e do mundo ${ }^{(14)}$.

O autor concebeu o sistema de crenças comparado a um átomo, sendo que, no centro desse sistema, estariam aquelas crenças imutáveis que formam, de fato, a versão do eu e do mundo. Na periferia do sistema, situam-se crenças pouco importantes e passíveis de mudanças. Para o autor, existem cinco tipos de crenças distribuídas numa dimensão central e periférica: 1) Tipo A: crenças preventivas (de consenso unânime) - são aquelas aprendidas por contato direto com o objeto da crença e são reforçadas por um consenso unânime, entre a pessoa e seus pares; 2) Tipo B: crenças primitivas (de consenso zero) - também aprendidas por contato direto, mas são particulares, envolvendo percepções pessoais, subjetivas e não dependendo do consenso unânime; 3) Tipo C: crenças de autoridade - são aquelas crenças que possuímos sobre em quais autoridades confiar ou não. São menos centrais do que as crenças primitivas e mudam de tempos em tempos; 4) Tipo D: crenças derivadas são aquelas que recebemos das fontes em que confiamos e com as quais nos identificamos; 5) Tipo E: crenças inconseqüentes - situadas na periferia do sistema de crenças, são arbitrárias, variáveis e mais facilmente passíveis de mudança ${ }^{(14)}$.

\section{METODOLOGIA}

Trata-se de um estudo descritivo exploratório, feito com 56 pacientes com doença coronariana, internados em um hospital de grande porte, realizado no período de outubro de 1998 a janeiro de 1999. Para obtenção dos dados, foi utilizado um instrumento semiestruturado, contendo perguntas abertas e fechadas, aplicado após consentimento formal do paciente e do comitê de ética em pesquisa, da Instituição. Esse instrumento continha dados de identificação dos sujeitos, tais como idade, sexo, escolaridade, renda familiar, diagnóstico médico, dados laboratoriais, e questões sobre o comportamento de fumar. Os relatos obtidos foram categorizados de acordo com o referencial, quanto à centralidade/perifericidade e adesão ou não às recomendações médicas de não fumar.

\section{APRESENTAÇÃO DOS DADOS E DISCUSSÃO}

A partir das categorizações, foi possível observar e descrever as crenças sobre o comportamento de fumar, contidas nas expressões verbais dos entrevistados. Podemos constatar maior concentração das crenças primitivas de consenso zero (31 emissões), seguidas das derivadas (13 emissões), das crenças de autoridade (14 emissões) e das crenças de consenso 100\% (3 emissões) e 1 inconseqüente $(E)$.

O critério adotado para considerar que um indivíduo apresentou adesão ao controle do fator de risco foi o seu relato verbal durante o período da coleta dos dados.

Passaremos a exemplificar as crenças contidas em cada tipo identificado, seguindo o sentido da centralidade para a periferia, conforme o sistema de crença adotado.

Crenças primitivas de consenso $100 \%$ sobre 0 comportamento de fumar

As crenças primitivas de consenso $100 \%$ sobre o comportamento de fumar foram encontradas em 3 relatos cujos entrevistados não possuem o fator de risco tabagismo. Eles acreditam que o fumo prejudica as artérias e os pulmões, portanto apresentam o comportamento de não fumar.

Crenças de consenso zero sobre o comportamento de fumar

Foram 31 entrevistados que apresentaram crença do tipo $B$, sendo que 25 não apresentaram comportamento de adesão à terapêutica indicada (ou seja, parar de fumar). 
Muitas crenças reforçam este tipo de comportamento:

Fumo porque não faz mal para o coração (E 15).

Fumo porque muita gente que não fuma, tem problema de coração (E 26, 50).

Tais relatos demonstram que as crenças em relação ao tabaco parecem estar desvinculadas dos malefícios que o fumo causa, embora ele tenha sido reconhecido por alguns como um risco:

Eu acho que o cigarro pode prejudicar a saúde, mas é uma coisa que eu não consigo largar (E 55).

Acho que a fumaça faz mal para os pulmões, para o coração não (E 50).

Quanto mais fumo, mais ataca o coração, mas eu não deixo de fumar (E 5).

Muitas intervenções dos profissionais da área de saúde estão alicerçadas na falta de conhecimento que 0 indivíduo possui a respeito do que causou o aparecimento de determinada doença. Com relação ao fator de risco "tabaco", inúmeros trabalhos demonstraram a sua importância na gênese das doenças cardiovasculares, e, embora esse fator de risco seja passível de modificação, parece que os serviços de saúde públicos ou privados, embora venham fornecendo essas informações sobre os malefícios desse comportamento "fumar", não tem alcançado muito efeito esperado:

Fumo desde os 5 anos de idade, os médicos dizem que foi ele, mas eu não acredito, no meu caso foi devido ao sol quente, os médicos estão errados (E 46).

Parei de fumar e acho que não valeu muito, porque infartei de novo, agora eu voltei a fumar (E 46).

Há relatos dos entrevistados que sugerem que os fumantes de longa duração e de grande número de cigarros fumados possuem menor probabilidade de tentar deixar de fumar, muitos pelo prazer "compensatório" que o tabagismo traz:

Fumo há 40 anos, eu encaro o cigarro como uma terapia, o fato de tentar ficar sem fumar me deixava nervoso, e com o cigarro voltou tudo a ficar certo (E 36).

Fumo desde os 9 anos de idade, fumo 3 maços por dia, acho que fiz uma coisa errada comigo. Mas o fumo me distrai (E 33).

Neste estudo foi observado que o cigarro está associado a um desejo/necessidade de alterar humor, acalmar, trazer bem estar em situações nas quais 0 estresse familiar, pessoal e no trabalho, gera desconforto.

Verificou-se, também, a utilidade dos cigarros para acalmar o indivíduo diante de situações de estresse, e esse achado também foi mencionado em alguns estudos $^{(15)}$. Em outros, verificou-se que existem algumas situações psicológicas do indivíduo, as quais são conscientes e inconscientes com relação à dependência do tabagista. As conscientes podem ser identificadas como estimulação, ritual, prazer, redução da tensão, hábito, vício, e, em nível inconsciente, o ato de fumar está associado ao status, segurança, "muleta social", uma companhia em situações de solidão ${ }^{(12)}$ :

O fumo me distrai quando estou nervoso; Ele me distrai e acalma o meu coração (E 25, 3, 23, 40, 44).

Nesse sentido, as crenças, como as do tipo B, de dimensão central, são originadas de experiências pessoais e externalizadas por meio de comportamentos nos quais é possível identificar manifestações de autoconceitos positivos e negativos. Os positivos constituem aqueles conceitos que guiariam as nossas aspirações e ambições, fazendo-nos acreditar que somos competentes, inteligentes, racionais, bondosos, etc.

Os negativos são aqueles relacionados com a nossa auto-imagem negativa, ou seja: as fobias, as complusões, as dúvidas, as ansiedades, a identidade, autocompetência, dentre outros:

(...) eu acho que estou viciado. Acho que o cigarro pode prejudicar a saúde, mas eu não consigo deixar de fumar (E 55).

Alguns sujeitos ainda atribuíram o aparecimento da doença coronariana a uma coisa predestinada, uma fatalidade:

Quando tem que acontecer, não adianta a pessoa segurar ou se esforçar, na vida as coisas acontecem (E 28) ou Quando a gente tem que passar por uma coisa, não é o cigarro que vai trazer (E 24).

A doença é vista como um acontecimento pelo qual a pessoa tem que passar, e não é evitada ou prevenida, excluindo-se os fatores de risco que predisporiam o indivíduo a apresentar tal doença (infarto).

Dos indivíduos que possuem o comportamento de não fumar (6), suas crenças indicaram que eles acreditam que iriam sobreviver menos, o fumo deixa o coração preto, e, não fumando, estão ajudando sua saúde. Existe a crença de valorização da vida e da qualidade de vida, uma vida mais saudável, com saúde, sugerindo que o fumo traz consigo a doença.

Crenças de autoridade sobre o comportamento de fumar

As crenças de autoridade (tipo C) foram 
identificadas nos relatos de 8 entrevistados sobre 0 comportamento de fumar, sendo 6 eram tabagistas. As crenças tipo $C$ estão relacionadas às crenças que possuímos sobre em qual autoridade confiar ou não. Por isso, a pessoa necessita criar um vínculo de identificação com determinada autoridade, que pode ser um irmão, um parente, um vizinho, um enfermeiro, um médico, etc, para que tais pessoas possam auxiliar o indivíduo naquilo em que deverão acreditar ou não.

A figura da autoridade mais referida neste estudo foi a do médico. Não houve nenhuma referencia com relação à enfermagem ou outras pessoas do convívio dos entrevistados. Gostaríamos de lembrar que desenvolvemos crenças sobre quais autoridades são positivas e quais são negativas, ou sobre em quais informações de tais autoridades confiamos, por serem verdadeiras:

Positiva:

Parei de fumar desde o infarto, acho que melhorei a tosse. O médico pediu para parar. (E 1, 20, 21, 22).

Parei de fumar devido à pressão alta, o médico sempre pediu. Talvez, se eu estivesse fumando, teria tido esse infarto antes (E 52).

Negativa:

Fumo há 29 anos um maço por dia. O médico sempre falou para eu deixar de fumar devido ao problema da pressão, mas eu nunca acreditei muito nele, porque ele tinha um maço no bolso, aí eu achava que não devia ser sério o que ele me falava (E 35).

Tenho dúvidas se o cigarro melhora mesmo o coração, porque tenho amigos que nunca fumaram e estão com problemas no coração (E 17).

Crenças derivadas sobre o comportamento de fumar

As crenças derivadas sobre o comportamento de fumar originarias de 13 pacientes, sendo que 7 tiveram crenças que favoreceram a adesão à recomendação de deixar de fumar.

A maioria das crenças tipo $D$ está relacionada à identificação do entrevistado com a autoridade divina, a religião:

(...) entreguei meu vício a Jesus Cristo, e ele me ajudou a parar de fumar (E 10).

A "provação" foi outra forma descrita:

Fumei 55 anos, estava viciado, parei porque tive uma provação: o cigarro estava fechando a minha garganta (E 18).

As crenças $D$ também se identificam com as informações oriundas da televisão ou derivadas de costume: na minha família mulher não fuma, eu escuto falar na televisão que o cigarro faz mal para o coração e eu dou muito valor a isso (E 34) ou do povo, da moda, ditada pela mídia da época:

Antigamente fumar representava um luxo para mim, era a moda da época. Todo rapaz tinha que ter um cigarro, senão não era homem, e isso era muito importante (E 36).

É importante observar como os veículos de comunicação de massa, muitas vezes, podem influenciar comportamentos de forma positiva, outras, negativas. Durante muito tempo, veicularam propagandas de cigarro que reforçavam a crença no belo, na virilidade $\mathrm{e}$ masculinidade, determinando um estilo de vida das pessoas. Recentemente, temos a ênfase, nos programas de televisão e artigos de revistas e jornais, dos malefícios que o cigarro traz.

Crenças inconseqüentes sobre o comportamento de fumar

As crenças do tipo E estão relacionadas à questão de gosto, preferências, e, embora sejam mais facilmente modificadas, elas também podem perdurar durante muito tempo dentro do sistema de crença de uma pessoa.

Fumo dois maços por dia, comecei a fumar porque eu achava bonito a pessoa segurar o cigarro(...) (E 55).

\section{CONSIDERAÇÕES FINAIS}

Este estudo possibilitou identificar as crenças segundo centralidade/perifericidade dos pacientes portadores de doença coronariana sobre o comportamento de fumar, ocorrendo maior concentração no sentido da centralidade das crenças, a saber: primitivas, de consenso zero (31 emissões), primitivas, de consenso 100\% ( 3 emissões), de autoridade (8 emissões) e derivadas (13 emissões).

Chamou-nos a atenção o fato de que a maioria das crenças relacionadas a comportamentos de não adesão (25 emissões) também se situaram na dimensão central do sistema de crenças, o que constitui elementos dificultadores aos profissionais da saúde, uma vez que uma dada crença pode estimular ou desestimular indivíduo a adotar práticas que promovam a sua própria saúde. E também o fato de estarem ligadas às experiências do eu, dependendo de um consenso externo, tornando-se muito mais difícil alterar determinados comportamentos.

O referencial em questão ${ }^{(14)}$ permitiu compreender que crenças situadas na periferia de um sistema de crenças 
(como as crenças derivadas) podem ser mais facilmente modificadas. Possibilitou, também, refletir sobre a maneira como vêm sendo veiculadas essas recomendações ou orientações para essa clientela, uma vez que elas têm-se mostrado pouco eficazes.
Sugerimos a inserção do modelo de crença ${ }^{(14)}$ na prática assistencial de enfermagem, para que se possam nortear os processos educativos valorizando-se as crenças existentes em cada comportamento expressado pelo indivíduo.

\section{REFERÊNCIAS BIBLIOGRÁFICAS}

1. Luepker VR. Epidemiologias das enfermidades aterosclerosas em grupos populacionais. Rocha: Ed. EPUC; 1997. p.1-10.

2. Lotufo PA. Epidemiologia das doenças cardíacas no Brasil: histórico, situação atual e proposta de modelo teórico. RSCESP 1996; 6(5):541-7.

3. Braunwald E. Os fatores de risco. In: Braunwald E. Tratado de medicina cardiovascular. 3aㅡ ed. Rio de Janeiro: Roca; 1991. p.1208-24.

4. Mancilha-Carvalho JJ. Antecedentes da doença coronária: os fatores de risco. Arq Bras Cardiol 1992; 58(4):263-7.

5. Kannel WB. Natural history cardiovascular risk. In: Holemberg NK. Hipertension: mechanisms and therapy. Philadelphia: Ed. Current Medicine; 1995. p.2-22.

6. Jorge PAR. Controle dos fatores de risco coronário depois do infarto do miocárdio - a disfunção endotelial e os eventos coronarianos. RSCESP 1995; 5(1):43-9.

7. Secretaria da Saúde do Estado de São Paulo (BR). Divisão de doenças crônicas não transmissíveis. São Paulo; 1992. 8. Dantas RAS. O perfil de pacientes com infarto agudo do miocárdio na perspectiva do modelo de campo de saúde. [Dissertação]. Ribeirão Preto (SP): Escola de Enfermagem de Ribeirão Preto/USP; 1996.

9. Trap-Jansen J. Effects of smoking on the heart and peripheral circulation. Am Heart J 1988; 115:263-7.

10. Vitola D. Fatores de risco coronariano. Arq Bras Cardiol 1994; 63(5):435-6.

11. Issa JS, Francisco YA. Obesidade e doenças cardíacas: aspectos epidemiológicos e preventivos. RSCESP 1996; 6(5):558-63.

12. Araújo AA Neto, Rassi RH, Labbadia EM. Tabagismo e doenças cardiovasculares. In: Porto CC. Doenças do coração, prevenção e tratamento: Rio de Janeiro: Guanabara Koogan; 1998. p.133-41

13. Candeias NMF, Marcondes RS. Diagnóstico em educação em saúde: um modelo para analisar as relações entre atitudes e práticas na área da saúde pública. Rev Saúde Pública 1979; 13:63-8.

14. Rokeach M. Crenças, atitudes e valores, uma teoria de organização e mudança. Rio de Janeiro: Interciência; 1981. 